\section{Confiabilidade dos dados antropométricos obtidos em crianças atendidas na Rede Básica de Saúde de Alagoas',ı}

\author{
Reliability of anthropometric data \\ obtained in children seen at the \\ Primary Public Healthcare Service \\ Network in Alagoas, Brazil
}

\author{
Maria Amália de Alencar Lima \\ Maria Alice Araújo Oliveira \\ Haroldo da Silva Ferreira \\ Faculdade de Nutrição da Universidade Federal de Alagoas.
}

' Pesquisa realizada com recursos financeiros da Secretaria de Estado da Saúde de Alagoas e do DECIT-MS/CNPq/SESAU-AL/FAPEAL (Processo PPSUS 013/2004).

" Artigo baseado na Dissertação de Mestrado de MAA Lima, apresentada à Faculdade de Nutrição da Universidade Federal de Alagoas em 2008.

Correspondência: Haroldo da Silva Ferreira. Av Pilar, 550, Cruz das Almas, Maceió/AL - 57038-430.

E-mail: haroldo.ufal@gmail.com

\section{Resumo}

Introdução: O monitoramento do crescimento infantil constitui-se em importante ferramenta para a construção de indicadores úteis ao planejamento de políticas e ações de atenção à saúde da criança. Para isso é necessário que os dados antropométricos obtidos apresentem satisfatória confiabilidade. Objetivo: Investigar a confiabilidade dos dados antropométricos produzidos nos Estabelecimentos de Assistência à Saúde (EAS) do SUS em Alagoas. Métodos: Para composição da amostra, sortearam-se 20 dentre os 102 municípios do Estado. Em seguida, dois EAS por município e, nestes, cerca de dez crianças menores de cinco anos. A amostra foi constituída de 40 EAS e 347 crianças. As medidas antropométricas (peso e estatura) foram aferidas (1) na rotina do serviço (S); (2) pelo pesquisador utilizando equipamento padrão (P) e; (3) pesquisador usando equipamentos do serviço (PS). Resultados: A aplicação do teste Kappa indicou concordância "substancial" $(K=$ $0,69)$ nas classificações de peso-para-idade (PI) entre S e P e "quase perfeita" $(\mathrm{K}=0,83)$ entre PS e P. Quanto à altura-para-idade (AI), a concordância entre $\mathrm{S}$ e $\mathrm{P}$ foi "discreta" (K $=0,27)$, passando a "moderada" $(K=0,56)$ entre PS e P. O Erro Técnico da Medição segundo faixas etárias $(</ \geq 24$ meses) indicou problemas na técnica e nos equipamentos. As medidas de peso corporal obtidas em S foram sistematicamente superestimadas ( $\mathrm{p}$ $<0,05$; teste dos sinais), o que determinou baixa sensibilidade e uma elevada taxa de falsos negativos (38,5\% para PI e $57,1 \%$ para AI). Conclusão: A baixa confiabilidade observada para a variável altura, desaconselha sua utilização na construção de indicadores de saúde até que os EAS sejam dotados de estadiômetros adequados e pessoal qualificado a utilizá-los. Quanto à variável peso, embora possa ser utilizada, é necessário melhorar sua qualidade por meio da capacitação dos antropometristas e da aquisição e/ou manutenção dos equipamentos.

Palavras-chave: Antropometria. Vigilância Nutricional. Sistemas de Informação. Confiabilidade. Erro Técnico na Medição. 


\section{Abstract}

Introduction: Monitoring child growth is a very important tool not only to build useful indicators for the evaluation and planning of public policies, but also for appropriate care to child health. In order to accomplish that anthropometric data from services need to present satisfactory reliability. Objective: To investigate the reliability of weight and height variables obtained from children at public healthcare services (PHS) of Alagoas. Methods: To build the sample, 20 municipalities were drawn among the 102 of the state. Then, two PHS were drawn per municipality, and in these, about ten children under five years. The final sample consisted of 40 PHS and 347 children. Anthropometric measures (weight and height) were taken (1) during routine service (S); (2) by the researcher using standard equipment (R), and (3) by the researcher using service equipment (RS). Results: The Kappa test indicated a "substantial" level of agreement $(\mathrm{K}=0.69)$ in the classifications of weightfor-age (WA) between S and R and "almost perfect” ( $K=0.83)$ in those between RS and R. Regarding height-for-age (HA), the level of agreement between $\mathrm{S}$ and $\mathrm{R}$ was "fair" $(\mathrm{K}$ $=0.27)$, to "moderate" $(\mathrm{K}=0.56)$ between RS and R. A Technical Measurement Error according to age groups $(<1 \geq 24$ months) indicated problems in the technique and equipment. Measurements of body weight obtained in PHS routine were systematically overestimated $(\mathrm{p}<0.05$; sign test), leading to low sensitivity, high specificity and a high rate of false negatives $(38.5 \%$ for WA and $57.1 \%$ for HA). Conclusion: The low reliability observed for the height variable cautions against its utilization to build health indicators, until the scenario can be improved. The weight variable may be used, but its quality must be improved by training anthropometrists, and through the acquisition and maintenance of equipment.

Keywords: Anthropometry. Nutritional Surveillance. Information Systems. Reliability. Technical Measurement Error.

\section{Introdução}

A concepção de Sistema de Vigilância Alimentar e Nutricional (SISVAN), oriunda da Conferência Mundial de Alimentação promovida pela FAO (Food and Agriculture Organization) em 1974, pressupõe um sistema de análise de informações de diversos setores, tais como agricultura, economia e saúde, tendo como finalidade promover periodicamente o diagnóstico descritivo e analítico da situação alimentar e nutricional da população, visando subsidiar o planejamento e a análise dos efeitos de políticas e programas, assim como a predição de tendências futuras. No nível nacional, a vigilância nutricional foi incluída no campo de atuação do Sistema Único de Saúde (SUS) por meio da Lei Orgânica do SUS ${ }^{1}$ que estabeleceu o monitoramento do perfil nutricional do grupo materno-infantil, estimulando a organização da produção de dados antropométricos nos centros e postos de saúde ${ }^{2}$.

A Política Nacional de Alimentação e Nutrição (PNAN), aprovada em 1999, incorporou entre suas diretrizes o monitoramento da situação alimentar e nutricional dos usuários do SUS, especialmente crianças e gestantes. A partir de então, tal atividade passou a fazer parte das rotinas de atendimento no âmbito da atenção básica de saúde, visando à detecção das situações de risco e a prescrição de ações de prevenção e controle ${ }^{3}$. No contexto da segurança alimentar e nutricional, o SISVAN figura como estratégia fundamental entre as diretrizes do Sistema Nacional de Segurança Alimentar e Nutricional ${ }^{4}$, podendo contribuir para o monitoramento da situação alimentar e nutricional, visando subsidiar o ciclo de gestão das políticas para a área nas diferentes esferas de governo.

O monitoramento da saúde da criança é proposto pelo Ministério da Saúde para avaliar e acompanhar, de maneira sistemática, os processos de crescimento e desenvolvimento infantil. Os índices antropométricos são utilizados como o principal critério desse acompanhamento ${ }^{5}$. Para isto, as variáveis antropométricas obtidas deveriam apresentar satisfatória confiabilidade ${ }^{6}$, uma 
vez que as medidas de peso e estatura estão sujeitas a várias fontes de erros. Estes, ainda que pequenos, podem determinar sensíveis distorções no diagnóstico nutricional ${ }^{7}$. Diante disso, é necessário que o pessoal envolvido na aferição dessas medidas esteja devidamente capacitado e motivado para a realização da tarefa ${ }^{8}$, bem como que os serviços disponham de equipamentos de qualidade satisfatória.

Diante do exposto, objetivou-se investigar se os dados antropométricos produzidos na rede básica de saúde do SUS em Alagoas apresentam confiabilidade satisfatória, constituindo-se em informações fidedignas que reflitam a realidade do perfil nutricional da população de crianças menores de cinco anos usuária da rede.

\section{Métodos}

\section{Planejamento amostral}

Em virtude dos recursos financeiros disponíveis para o projeto, bem como por critérios operacionais, trabalhou-se com uma amostra de conveniência formada por 40 Estabelecimentos de Assistência à Saúde (EAS). Para garantir a randomização do processo de seleção, foram sorteados 20 dentre os 102 municípios alagoanos (amostragem sistemática com probabilidade proporcional ao número de habitantes). Posteriormente, sortearam-se dois EAS em cada município. Num terceiro estágio, foram alocadas dez crianças menores de cinco anos por EAS. Para o sorteio dos EAS levou-se em consideração sua inscrição no Cadastro Nacional de Estabelecimentos de Saúde do Ministério da Saúde ${ }^{9}$ que, à época, totalizava 189 unidades. Portanto, o plano amostral previu a inclusão de 400 crianças oriundas de 40 EAS (40/189) de 20 municípios (20/102).

\section{Coleta de dados}

A coleta de dados foi realizada no período de março a julho de 2006. Em cada EAS foram avaliadas as primeiras 10 crianças menores de cinco anos que compareceram ao serviço e cujo acompanhante (mãe ou responsável) ofereceu o consentimento. Crianças portadoras de alterações anatômicas que impedissem a avaliação antropométrica (deformidades físicas, doenças genéticas, edema) não foram incluídas.

Em 19 EAS não houve número suficiente de crianças na faixa etária alvo, o que, ao lado das recusas e exclusões, determinou que a amostra final ficasse constituída por 347 crianças. Não foi possível a recuperação dessas perdas porque, de acordo com o planejamento operacional estabelecido, os pesquisadores cumpriam um cronograma que determinava apenas uma visita a cada EAS (um expediente de trabalho).

Cada criança incluída no estudo teve suas medidas antropométricas aferidas em três situações, possibilitando comparações inter-examinadores e inter-equipamentos:

- pelo funcionário da EAS que realizava a pré-consulta, ocasião em que se executou a antropometria (neste trabalho, este servidor será designado como antropometrista, independentemente de sua qualificação profissional). Essa aferição foi procedida na rotina do serviço sem qualquer interferência externa;

- pelos pesquisadores, utilizando equipamento próprio e de adequada confiabilidade (medida padrão-ouro). Essa etapa foi realizada em momento posterior ao atendimento da criança, de modo a evitar que a observação dos procedimentos empregados pelos pesquisadores interferisse na forma de atuação dos antropometristas;

- novamente pelos pesquisadores, porém utilizando o mesmo equipamento usado pelo antropometrista do serviço. Para complementar a avaliação das crianças, verificou-se com a mãe ou responsável a posse do Cartão da Criança e se o portava no momento. Esse procedimento foi necessário em virtude da importância da utilização adequada desse instrumento em relação aos objetivos do SISVAN ${ }^{8}$.

Para o treinamento e padronização da equipe de pesquisadores, formada por uma 
nutricionista e três estudantes de Nutrição, adotaram-se as recomendações do Ministério da Saúde ${ }^{5}$, as mesmas que deveriam ser utilizadas nos EAS $^{5}$.

Entre os equipamentos da equipe constava:

- balança eletrônica portátil (Marte PP180, São Paulo, Brasil) com capacidade para $180 \mathrm{~kg}$ e sensibilidade de $0,1 \mathrm{~kg}$, calibrada pelo Instituto de Metrologia e Qualidade de Alagoas e examinada diariamente contra peso padrão $(5 \mathrm{~kg})$;

- estadiômetro horizontal em madeira para aferição do comprimento das crianças menores de 24 meses em posição de decúbito dorsal;

- estadiômetro vertical em madeira para aferição da altura das crianças com idade igual ou maior que 24 meses em posição ortostática. Tanto o estadiômetro horizontal quanto o vertical eram dotados de fita métrica inextensível (fibra de vidro), com sensibilidade para $0,1 \mathrm{~cm}$. Neste trabalho os termos peso e massa corporal serão utilizados como sinônimos, o mesmo ocorrendo em relação às palavras altura e estatura.

Antes da coleta de dados, foi feito contato com os diretores de cada EAS para lhes explicitar detalhes do estudo, solicitar permissão para realizá-lo e informações relativa ao cronograma de atendimento na Unidade, além de definir o fluxo do trabalho a ser realizado pelos pesquisadores de modo a garantir o mínimo de interferência na rotina do serviço.

Para avaliar a adequação dos procedimentos técnicos realizados pelo antropometrista, um pesquisador o observava enquanto realizava as medidas. Para isto, utilizou-se um roteiro padronizado de acordo com as Normas Técnicas de Antropometria para a Vigilância Alimentar e Nutricional ${ }^{5}$. Para que não modificasse seu procedimento de rotina, o antropometrista era informado da pesquisa só após a finalização da aferição das medidas de todas as crianças arroladas para o estudo no respectivo EAS. Nessa ocasião, além da explicitação dos objetivos e procedimentos da pesquisa, solicitava-se sua permissão para inclusão no estudo, a qual era expressa através de sua assinatura no Termo de Consentimento Livre e Esclarecido. Em seguida, o mesmo era entrevistado com vistas a possibilitar a caracterização do perfil dos recursos humanos dos EAS envolvidos na produção dos dados antropométricos. No formulário utilizado constavam questões sobre sexo, idade, escolaridade, formação profissional, situação funcional e capacitação para a antropometria.

Os valores das medidas antropométricas aferidas pelos antropometristas eram coletados diretamente dos prontuários, após o atendimento médico procedido na rotina do EAS. A idade da criança foi calculada a partir da data de seu nascimento registrada no Cartão da Criança ou no prontuário. $\mathrm{Na}$ última etapa da seqüência da coleta de dados, os espaços físicos dos EAS destinados à antropometria eram analisados quanto às suas características e às dos equipamentos/ instrumentos existentes, dos quais eram verificados o posicionamento, funcionamento e estado de conservação. Por falta de critérios objetivos, essa análise foi procedida de forma qualitativa por um único profissional com experiência em estruturação e supervisão de serviços de antropometria.

O fluxo do trabalho de campo e todos os instrumentos elaborados para a coleta de dados foram previamente testados em estudo piloto, o qual se realizou em um EAS do município de Maceió não selecionado para o estudo. Na calibração dos entrevistadores, no que diz respeito à antropometria, adotou-se o procedimento proposto por Habicht ${ }^{10}$.

\section{Análises estatísticas}

O programa Epi-Info 6.04 (Centers for Disease Control and Prevention, Atlanta, Georgia, USA) foi utilizado para digitação em dupla entrada independente, validação e tabulação dos dados antropométricos. O processo de validação teve por finalidade excluir apenas erros de digitação, pois, 
de acordo com os objetivos do trabalho, considerou-se inadequada a exclusão de possíveis outliers.

Para classificação do estado nutricional das crianças foram utilizados os índices peso-para-idade (PI) e estatura-para-idade (EI), expressos em percentis (p) em relação à curva de referência do National Center for Health Statistics ${ }^{11}$, o mesmo utilizado pelo SISVAN $^{5}$ à época da coleta de dados. Para tanto, foram considerados os pontos de corte adotados pelo Ministério da Saúde ${ }^{5}$, que estabelece as seguintes categorias para o índice PI: peso baixo para a idade $(<\mathrm{p} 3)$, risco nutricional ( $\geq$ p3 e $<$ p10), eutrofia ( $\geq$ p10 e $<$ p97) e sobrepeso ( $\geq$ p97), e para o índice AI: déficit de altura para a idade $(<\mathrm{p} 3)$, risco nutricional $(\geq \mathrm{p} 3 \mathrm{e}<\mathrm{p} 10) \mathrm{e}$ crescimento adequado ( $\geq$ p10). Embora não fosse objetivo deste estudo proceder à classificação do estado nutricional das crianças, esse procedimento foi necessário visando comparar a concordância entre as classificações geradas nas três situações em que as variáveis peso e estatura foram obtidas.

Para se conhecer o grau de concordância entre as classificações nutricionais obtidas nas três situações estudadas calculou-se o Coeficiente Kappa de Cohen. Para interpretação da concordância usou-se o critério de Landis \& $\mathrm{Koch}^{12}$ : insignificante $(0,00-0,20)$; discreta $(0,21-0,40)$; moderada $(0,41-0,60)$; substancial $(0,61-0,80)$; quase perfeita $(0,81-1,00)$.

Visando-se definir a precisão das medidas obtidas, calculou-se o Erro Técnico da Medida (ETM), através dos seguintes procedimentos $^{13}$ :

- Cálculo do desvio entre os pares de medidas $(\mathrm{d}=$ medida situação 1 - medida situação 2);

- Elevação dos desvios ao quadrado $\left(\mathrm{d}^{2}\right)$;

- Somatório dos $\mathrm{d}^{2}\left(\sum \mathrm{d}^{2}\right)$

- Cálculo do ETM absoluto:

ETM absoluto $=\frac{\sqrt{\Sigma} \mathrm{d}^{2}}{2 \mathrm{n}}$

onde $\mathrm{n}$ = número de observações;
- Cálculo dovalor médio da variável(VMV):

a. Cálculo da média dos pares em comparação (medida situação 1 + medida situação 2) /2;

b. Somatório das médias e divisão pelo número de pares.

- Cálculo do ETM relativo:

ETM relativo $=\frac{\mathrm{ETM}}{\mathrm{VMV}} \times 100$

Valores de ETM relativos iguais ou menores que 2,0 foram considerados aceitáveis, enquanto aqueles superiores a esse ponto de corte foram classificados como inaceitáveis, conforme proposto por Pederson \& Gore ${ }^{14}$.

Para avaliar a validade das classificações obtidas segundo as diferentes situações em que os dados antropométricos foram obtidos, calcularam-se a sensibilidade, a especificidade, o valor preditivo positivo, o valor preditivo negativo e as respectivas taxas de falsos positivos e falsos negativos, tendo-se como "padrão-ouro" a classificação obtida pelos pesquisadores com seus equipamentos. Para isso, os dados foram organizados em uma tabela de contingência e analisados conforme descrito no Quadro $1^{15}$ :

Parâmetros calculados:

Sensibilidade (A/A+C.100): Proporção de indivíduos desnutridos que são corretamente identificados;

Especificidade (D/B + D.100): Proporção de indivíduos eutróficos que são assim diagnosticados;

Valor preditivo positivo (A/A + B.100): probabilidade de ser realmente desnutrido dado que o procedimento diagnóstico atribuiu tal resultado;

Valor preditivo negativo (D/C $+\mathrm{D} .100)$ : probabilidade de não se ter desnutrição dado que o procedimento diagnóstico atribuiu tal resultado;

Taxa de falsos positivos (B/B + D.100): proporção de indivíduos eutróficos que foram equivocadamente diagnosticados como desnutridos;

Taxa de falsos negativos (C/A + C.100): proporção de indivíduos desnutridos que foram de forma equivocada diagnosticados como eutróficos. 
Quadro 1 - Matriz para os cálculos dos parâmetros de validade diagnóstica das classificações obtidas a partir dos dados de peso e estatura coletados nos Estabelecimentos de Assistência à Saúde do SUS no estado de Alagoas.

Chart 1 - Matrix for calculating diagnostic validity parameters of the classifications obtained from weight and height data collected in SUS Healthcare Facilities in the state of Alagoas.

\begin{tabular}{|c|c|c|c|}
\hline \multirow{2}{*}{$\begin{array}{l}\text { Classificação S } \\
\text { ou classificação PS }\end{array}$} & \multicolumn{2}{|c|}{ Classificação P (padrão-ouro) } & \multirow{2}{*}{ TOTAL } \\
\hline & Desnutrido & Normal & \\
\hline Desnutrido & $\begin{array}{c}\text { A } \\
\text { (Verdadeiros positivos) }\end{array}$ & $\begin{array}{c}\text { B } \\
\text { (Falsos positivos) }\end{array}$ & $A+B$ \\
\hline Normal & $\begin{array}{c}\text { C } \\
\text { (Falsos negativos) }\end{array}$ & $\begin{array}{c}\mathrm{D} \\
\text { (Verdadeiros negativos) }\end{array}$ & $C+D$ \\
\hline TOTAL & $A+C$ & $B+D$ & $A+B+C+D$ \\
\hline
\end{tabular}

Classificação P:Elaborada a partir de dados coletados pelo pesquisador com equipamento próprio (de alta confiabilidade);

Classificação S: Elaborada a partir de dados coletados na rotina do serviço de saúde;

Classificação PS: Elaborada a partir de dados coletados pelo pesquisador, porém usando os equipamentos do serviço de saúde.

$P$ Classification = data obtained by the researcher using standard equipment.

SClassification = data obtained by the professional of the health care unit in routine service.

PS Classification = data obtained by the researcher using service equipment.

Para identificar um possível viés na obtenção das medidas e a direção do erro, calcularam-se as diferenças entre as medidas obtidas nas condições avaliadas (antropometrista ou pesquisador com equipamento do EAS) e as obtidas na condição padrão. Em seguida, verificou-se a existência de predominância de sinais positivos ou negativos. A significância estatística dos resultados foi analisada por meio do teste dos sinais.

\section{Aspectos éticos}

O trabalho foi aprovado pelo Comitê de Ética em Pesquisa da Universidade Federal de Alagoas como parte do projeto "Diagnóstico de saúde da população maternoinfantil do Estado de Alagoas" (Processo n. ${ }^{\circ}$ 010102/2003-35).

Os funcionários dos EAS e as mães ou responsáveis pelas crianças foram informados sobre a finalidade da pesquisa e, em caso de aceitação, assinaram o Termo de Consentimento Livre e Esclarecido, sendo garantida a confidencialidade das informações.

\section{Resultados}

Dentre os 40 EAS sorteados para a amostra, verificou-se que a maioria $(77,5 \%)$ estava localizada em área urbana dos municípios e que 70,0\% adotavam a estratégia Saúde da Família (ESF) como modelo de organização da Atenção Básica.

Foram avaliadas nessas 40 EAS 347 crianças menores de cinco anos, sendo que a maior parte encontrava-se nos dois primeiros anos de vida $(61,6 \%)$.

A quase totalidade das mães $(98,9 \%)$ referiu possuir o Cartão da Criança, porém $27,5 \%$ delas não o portavam no momento da consulta.

Em praticamente todos os EAS encontrou-se balança antropométrica mecânica de plataforma $(97,5 \%)$ no local de realização das medidas, porém $48,7 \%$ apresentavam estado de conservação insatisfatório e 35,9\% estavam posicionadas de forma inadequada no ambiente. Quanto à balança pediátrica mecânica, foi encontrada em $85,0 \%$ dos EAS. A maioria delas $(73,5 \%)$ estava em precárias condições de conservação e $26,5 \%$ posicionadas de forma inadequada no ambiente. Foram encontradas, ainda, balança do tipo pessoal mecânica portátil (12,5\%) e balança mecânica de suspensão tipo gancho (2,5\%).

Em relação aos instrumentos utilizados na aferição do comprimento/altura, os tipos mais comuns nos serviços de saúde foram: estadiômetro horizontal em madeira $(27,5 \%)$, dos quais $36,4 \%$ apresentavam 
estado de conservação insatisfatório, e fita métrica $(25,0 \%)$ afixada na parede, porém posicionada de forma inadequada em todos os EAS.

Foi observado que $40,0 \%$ dos EAS visitados dispunham de local adequado para a realização da antropometria. Nos demais, não havia local específico, de modo que a coleta de dados ocorria nas salas de curativos, vacinação ou sala de espera e, ainda, nos corredores do serviço de saúde.

Um percentual de $90 \%$ dos servidores era do sexo feminino e $48 \%$ apresentavam idades entre 30 e 39 anos. Quanto à escolaridade, $59 \%$ completaram o ensino médio. Os servidores que exerciam suas funções em caráter efetivo representavam $85 \%$. Em relação ao cargo ocupado, 86\% eram Auxiliares de Enfermagem.

Nos últimos 24 meses apenas $18 \%$ dos profissionais participaram de capacitação em antropometria e $13 \%$ em manejo do Cartão da Criança. Apenas 15\% referiram seguir normas específicas para a coleta do peso e estatura. Todos os antropometristas informaram registrar os dados obtidos no prontuário da criança; todavia, o registro na ficha da Vigilância Nutricional e no Cartão da Criança foi relatado por apenas, respectivamente, $26 \%$ e $38 \%$ deles.

Em todos os EAS foi observada a realização da aferição da massa corporal das crianças. No entanto, a aferição da altura ocorreu em somente 87 crianças $(25,1 \%)$. Observou-se que as medidas eram sistematicamente efetuadas apenas pelo antropometrista, sem a ajuda da mãe ou de qualquer outra pessoa.

Observou-se que na maioria dos EAS (75\%), as crianças menores de 24 meses eram pesadas em balança pediátrica mecânica, porém a balança antropométrica de plataforma mecânica também foi utilizada para essa faixa etária $(21,1 \%)$. Na maior parte das EAS (85\%), as crianças com idade superior a 24 meses foram pesadas em balança antropométrica de plataforma mecânica. Três EAS (7,5\%) utilizavam balança pessoal mecânica portátil com graduação mínima de 1,0 kg. Observou-se que, em dois desses três EAS onde isso ocorreu, havia balança mecânica pediátrica ou de plataforma em boas condições de uso.

Entre os 15 EAS (37,5\%) que realizaram a tomada da medida do comprimento/altura havia uma diversidade de instrumentos e de graduações (subdivisões variando de 0,1 a $1,0 \mathrm{~cm}$ ). $\mathrm{O}$ estadiômetro horizontal em madeira contendo fita métrica com variação na graduação entre 0,1 e $0,5 \mathrm{~cm}$ foi encontrado em alguns EAS (26,7\%) para a aferição do comprimento das crianças menores de 24 meses e, de forma inadequada, das crianças com idade acima de 24 meses. Observou-se ainda que, em cinco dentre essas 15 EAS, foi utilizada a régua antropométrica que vem acoplada à balança de plataforma para a aferição da altura das crianças.

Na maioria dos casos, os antropometristas não seguiam as recomendações preconizadas pelo Ministério da Saúde para a aferição da massa corporal (Tabela 1). Dentre os erros mais freqüentes destacou-se a não calibração da balança antes de iniciar a pesagem dos menores de 24 meses $(93,5 \%$ em relação às 201 vezes em que o procedimento foi realizado). Apenas $15,9 \%$ das crianças foram despidas corretamente antes da aferição. Os antropometristas aguardaram o alinhamento da agulha do braço e o fiel da balança antes da leitura em somente metade das aferições realizadas.

A leitura do resultado obtido em balança pediátrica mecânica foi realizada de forma correta em apenas $44,6 \%$ dos casos. Esse procedimento melhorou consideravelmente $(90,3 \%)$ quando foi utilizada a balança de plataforma. Após a realização da leitura, o procedimento de retornar os cursores ao zero da escala numérica da balança foi realizado em $59,8 \%$ das medidas. O registro do valor da massa corporal foi feito após a leitura e diretamente no prontuário de 91,1\% das crianças, ao passo que a anotação também no Cartão da Criança ocorreu em apenas $13,3 \%$.

Analisando os procedimentos técnicos preconizados pelo Ministério da Saúde durante a tomada das medidas de comprimento/altura (Tabela 2), observou-se que 
Tabela 1 - Freqüências dos procedimentos da técnica para medir a massa corporal realizados em crianças menores de cinco anos atendidas na rede básica de saúde do SUS em Alagoas, 2006.

Table 1 - Frequency of appropriate procedures to measure body mass performed in children under five years seen at the primary public healthcare service network in Alagoas (Brazil), 2006.

\begin{tabular}{|c|c|c|c|c|c|c|c|c|c|}
\hline \multirow{3}{*}{ Procedimentos } & \multicolumn{6}{|c|}{ Faixa etária (meses) } & \multirow{2}{*}{\multicolumn{3}{|c|}{ Total }} \\
\hline & \multicolumn{3}{|c|}{$0-23,9$} & \multicolumn{3}{|c|}{$24-60$} & & & \\
\hline & $\mathrm{N}$ & $\mathrm{n}$ & $\%$ & $\mathrm{~N}$ & $\mathrm{~N}$ & $\%$ & $\mathrm{~N}$ & $\mathrm{n}$ & $\%$ \\
\hline Calibrou o fiel da balança & 201 & 13 & 6,5 & 125 & 0 & 0,0 & 326 & 13 & 4,0 \\
\hline Travou, após calibrar & 201 & 2 & 1,0 & 125 & 2 & 1,6 & 326 & 4 & 1,2 \\
\hline Esperou zerar o visor ${ }^{1}$ & 12 & 12 & 100,0 & 9 & 9 & 100,0 & 21 & 21 & 100,0 \\
\hline Despiu a criança & 213 & 40 & 18,8 & 134 & 15 & 11,2 & 347 & 55 & 15,9 \\
\hline $\begin{array}{l}\text { Esperou até alinhar a agulha do braço e o fiel } \\
\text { da balança }\end{array}$ & 201 & 91 & 45,3 & 125 & 73 & 58,4 & 326 & 164 & 50,3 \\
\hline Esperou fixar o valor da medida no visor ${ }^{1}$ & 12 & 12 & 100,0 & 9 & 9 & 100,0 & 21 & 21 & 100,0 \\
\hline $\begin{array}{l}\text { Leu o valor da medida de frente, no nível da } \\
\text { escala numérica }\end{array}$ & 213 & 95 & 44,6 & 134 & 121 & 90,3 & 347 & 216 & 62,2 \\
\hline Registrou o valor da medida no prontuário & 213 & 191 & 89,7 & 134 & 125 & 93,3 & 347 & 316 & 91,1 \\
\hline $\begin{array}{l}\text { Retornou os cursores ao zero na escala } \\
\text { numérica }\end{array}$ & 201 & 127 & 63,2 & 125 & 68 & 54,4 & 326 & 195 & 59,8 \\
\hline $\begin{array}{l}\text { Registrou o valor da medida no Cartão da } \\
\text { Criança }\end{array}$ & 213 & 33 & 15,5 & 134 & 13 & 9,7 & 347 & 46 & 13,3 \\
\hline
\end{tabular}

83,9\% das crianças estavam sem calçados e com a cabeça livre de adereços. O posicionamento adequado do corpo aconteceu em cerca $29,5 \%$ das crianças de 24 a 60 meses. O procedimento de conduzir a parte móvel do estadiômetro contra a cabeça, no caso da aferição da altura, ou contra os pés, quando aferindo o comprimento, foi realizado corretamente em $16,1 \%$ das crianças, principalmente pela ausência desse componente no equipamento ou de recurso similar (um esquadro, por exemplo). A leitura da medida foi realizada de modo adequado em apenas $44,8 \%$ dos casos e o respectivo registro feito logo após a leitura e diretamente no prontuário em $87,4 \%$ das aferições.

Na comparação entre as classificações nutricionais, segundo o índice peso-paraidade, produzidas pelo pesquisador no equipamento padrão em relação àquelas produzidas pelos antropometristas, verificou-se, de acordo com o coeficiente Kappa de Cohen, ter havido uma concordância substancial $(K=0,69 ; p<0,05)$. No entanto, quando nessa comparação se substituiu a classificação obtida no serviço por aquela realizada pelo pesquisador usando o equipamento do serviço, houve uma concordância quase perfeita $(K=0,83 ; p<0,05)$.

A concordância entre as classificações padrão e as obtidas pelos antropometristas em relação ao índice altura-para-idade foi considerada discreta $(K=0,27)$. A concordância passou a moderada $(K=0,56)$ quando se comparou os resultados produzidos pelo pesquisador no equipamento padrão com aqueles produzidos pelo pesquisador usando o equipamento do serviço.

Os resultados obtidos pelo cálculo do ETM (Tabela 3) revelam que, em todas as situações foi observada a classificação "inaceitável” (ETM > 2,0), exceto na comparação das medidas de estatura e massa corporal (esta só na faixa etária igual ou superior a 24 meses) obtidas pelo pesquisador com equipamento do serviço. 
Tabela 2 - Freqüências dos procedimentos da técnica para medir a estatura realizados em crianças menores de cinco anos atendidas na rede básica de saúde do SUS em Alagoas, 2006.

Table 2 - Frequency of appropriate procedures to measure height performed in children under five years seen at the primary public healthcare service network of Alagoas (Brazil), 2006.

\begin{tabular}{lcccccc}
\hline & \multicolumn{3}{c}{ Faixa etária (meses) } & \multirow{2}{*}{ Total (N=87) } \\
\cline { 2 - 5 } Procedimentos & $0-23,9(\mathrm{~N}=43)$ & $24-60(\mathrm{~N}=44)$ & \\
\cline { 2 - 5 } & $\mathrm{n}$ & $\%$ & $\mathrm{n}$ & $\%$ & $\mathrm{n}$ & $\%$ \\
\hline Deixou a criança descalça e sem adereços na cabeça & 38 & 88,4 & 35 & 79,5 & 73 & 83,9 \\
Colocou a criança no centro do equipamento & 20 & 46,5 & 25 & 56,8 & 45 & 51,7 \\
Posicionou o corpo da criança & 0 & 0,0 & 13 & 29,5 & 13 & 14,9 \\
Levou a parte móvel do equipamento até as plantas dos pés' ou & 2 & 4,7 & 12 & 27,3 & 14 & 16,1 \\
contra a cabeça & & & & & & \\
Leu o valor da medida quando esteve seguro de que a criança & 15 & 34,9 & 24 & 54,5 & 39 & 44,8 \\
não se moveu da posição adequada & & & & & & \\
Registrou o valor da medida no prontuário & 38 & 88,4 & 38 & 86,4 & 76 & 87,4 \\
\hline
\end{tabular}

$\mathrm{N}=$ número de crianças avaliadas $/ \mathrm{N}=$ number of children assessed.

$\mathrm{n}=$ número de vezes em que o procedimento foi observado $/ \mathrm{n}=$ number of times procedure was observed.

${ }^{1}$ Crianças $<24$ meses / ' Children $<24$ months.

${ }^{2}$ Crianças $\geq 24$ meses $/{ }^{2}$ Children $\geq 24$ months.

Tabela 3 - Erro Técnico da Medição (ETM) na aferição da massa corporal e do comprimento ou estatura de crianças menores de cinco anos atendidas na rede básica de saúde do SUS em Alagoas, segundo faixa etária e origem da obtenção da medida.

Table 3 - Technical measurement error (ETM\%) in the measurement of body mass and length or height of children under five years seen at the primary public healthcare service network in Alagoas (Brazil), according to age and origin of obtaining the measurement.

\begin{tabular}{lcccccc}
\hline Faixa etária & \multicolumn{3}{c}{ Massa corporal } \\
\cline { 2 - 7 } (meses) & S vs. PS & PS vs. $\mathbf{P}$ & S vs. $\mathbf{P}$ & S vs. PS & PS vs. P & S vs. P \\
\hline $0-23,9$ & 4,98 & 3,23 & 4,34 & 2,50 & $0,85^{*}$ & 2,62 \\
$24-60$ & 2,59 & $1,67^{*}$ & 2,26 & 2,52 & $0,78^{*}$ & 2,63 \\
Parâmetro & Técnica & Equipamento & Ambos & Técnica & Equipamento & Ambos \\
avaliado & & & & & & \\
\hline
\end{tabular}

$S=$ Medida obtida na rotina do serviço / $S=$ Measurements obtained in service routine.

PS = Medida obtida pelo pesquisador, utilizando o equipamento do serviço (o mesmo usado em "S" pelo antropometrista da unidade de saúde / PS = Measurement obtained by the researcher, using service equipment (the same used in " $S$ " by the healthcare service technician).

$\mathrm{P}=$ Medida obtida pelo pesquisador utilizando equipamento de alta confiabilidade (medida padrão-ouro) / $P=$ Measurement obtained by the researcher using high-reliability equipment (gold-standard measure).

* Classificação aceitável $($ ETM $<2,0) / *$ Acceptable classification $(E T M<2.0)$

Os resultados relativos aos testes de validade diagnóstica encontram-se expressos na Tabela 4. Verifica-se que, tanto nas classificações que envolveram o índice peso-para-idade, como naquelas baseadas no índice altura-para-idade, obteve-se alta especificidade e baixa sensibilidade. A sensibilidade da classificação obtida com os dados do pesquisador com equipamento do serviço foi superior àquela produzida com os dados do antropometrista $(88,5 \%$ vs. $61,1 \%$ em relação ao peso-para-idade; $50,0 \%$ vs. $42,9 \%$ em relação à altura-para-idade).

Considerando que a sensibilidade, na presente situação, diz respeito à capacidade do procedimento em identificar os verdadeiros desnutridos, e verificando-se que esta foi menor quando a classificação foi realizada com os dados da rotina do serviço, surgiu a necessidade de investigar a possi- 
Tabela 4 - Validade diagnóstica das classificações antropométricas obtidas em crianças menores de 5 anos atendidas na rede básica de saúde do SUS em Alagoas, segundo a origem da obtenção da medida.

Table 4 - Validity of the anthropometric classifications obtained in children under 5 years seen at the primary public healthcare service network in Alagoas (Brazil), according to origin of obtaining the measurement.

\begin{tabular}{lcccc}
\hline Parâmetro' (\%) & \multicolumn{2}{c}{ Peso-para-idade } & \multicolumn{2}{c}{ Altura-para-idade } \\
\cline { 2 - 5 } & PS & S & PS & S \\
\hline Sensibilidade & 88,5 & 61,5 & 50,0 & 42,9 \\
Especificidade & 98,7 & 98,1 & 95,7 & 94,9 \\
Valor preditivo positivo & 85,2 & 72,7 & 50,0 & 42,9 \\
Valor preditivo negativo & 99,0 & 96,9 & 95,7 & 94,9 \\
Taxa de falsos positivos & 1,3 & 1,9 & 4,3 & 5,1 \\
Taxa de falsos negativos & 11,5 & 38,5 & 50,0 & 57,1 \\
\hline
\end{tabular}

$\mathrm{PS}=$ Classificação obtida a partir de dados coletados pelo pesquisador, utilizando o equipamento do serviço (o mesmo usado em " $\mathrm{S}$ " pelo antropometrista da unidade de saúde) / PS = measurement obtained by the researcher, using service equipment (the same used in "S" by the healthcare service technician).

$\mathrm{S}=$ Classificação obtida a partir de dados coletados pelo servidor da Unidade na rotina do serviço $/ \mathrm{S}=$ measurement obtained in service routine.

${ }^{1}$ Calculados tendo como classificação de referência (padrão-ouro) aquela obtida pelo pesquisador utilizando equipamento próprio e de alta confiabilidade

$/{ }^{1}$ Calculated, taking as gold-standard the classification obtained by the researcher using high-reliability equipment.

bilidade de que os erros de aferição não tenham sido aleatórios, mas sim sistemáticos. Para isso, investigou-se por meio do teste dos sinais se havia uma predominância de erros em uma determinada direção. Para o índice peso-para-idade foram obtidos os seguintes resultados:

- Padrão-ouro vs. pesquisador com equipamento do serviço: 142 valores inferiores (-), 163 valores superiores (+) e 38 valores iguais (=); $\mathrm{p}=0,25$.

- Padrão-ouro vs. antropometrista com equipamento do serviço: 95 (-), 218 (+), $30(=) ; \mathrm{p}<0,001$.

Verifica-se que apenas na segunda comparação houve diferença estatisticamente significativa, indicando uma maior proporção de erros positivos, ou seja, uma tendência sistemática (viés) a superestimar o peso real da criança.

Para o índice altura-para-idade verificou-se entre as medidas obtidas pelo pesquisador com o equipamento do serviço uma maior proporção de valores inferiores em relação às obtidas pelo padrão-ouro. $\mathrm{Na}$ comparação com as medidas obtidas pelo antropometrista não foram observadas diferenças significativas:

- Padrão-ouro vs. pesquisador com equipamento do serviço: 55 (-), 28 (+) e 4 (=); $\mathrm{p}=0,01$.
- Padrão-ouro vs. antropometrista com equipamento do serviço: 50 (-), 35 (+), $2(=) ; \mathrm{p}=0,18$.

\section{Discussão}

A maior parte das crianças incluídas neste estudo encontrava-se nos dois primeiros anos de vida. Possivelmente esse fato decorreu da existência de uma programação para o acompanhamento de saúde no primeiro ano de vida, segundo um calendário mínimo de consultas agendadas pelos serviços, com oferta de ações básicas prioritárias, tais como imunização, prevenção e controle de doenças diarréicas e das infecções respiratórias agudas, promoção do aleitamento materno e monitoramento do crescimento e desenvolvimento ${ }^{6}$. Adicionalmente, nessa faixa etária as mães buscam mais os serviços de saúde em função da maior morbidade verificada nesse período ${ }^{16}$.

A quase totalidade das mães referiu possuir o Cartão da Criança, resultado semelhante aos encontrados por Batista Filho \& Romani ${ }^{17}$ e Veras et al. ${ }^{18}$. Quanto ao cargo ocupado pelos antropometristas, observou-se uma predominância de Auxiliares de Enfermagem, tal como encontrado por Carvalho et al. ${ }^{8}$ nos serviços de saúde de Pernambuco.

Os resultados apresentados evidencia- 
ram um baixo nível de investimento em atividades de capacitação em antropometria e utilização de normas técnicas, as quais são de grande importância para consulta em caso de dúvidas ${ }^{18}$.

A análise dos resultados referentes à qualidade da medida do comprimento/ estatura ficou comprometida em virtude do pequeno número de EAS onde esse procedimento era realizado. $\mathrm{Na}$ amostra estudada por Batista Filho \& Romani ${ }^{17}$, a proporção de crianças que tiveram sua estatura aferida foi de apenas $16,3 \%$.

Observou-se uma fração importante de crianças menores de dois anos $(21,1 \%)$ sendo pesada em balança antropométrica de plataforma mecânica, proporção bem acima daquela observada $(6,0 \%)$ por $\mathrm{Ca}$ pelli et al. ${ }^{19}$. Adicionalmente, em alguns EAS utilizava-se balança pessoal mecânica portátil com graduação mínima de $1,0 \mathrm{~kg}$, contrariando padrões e protocolos recomendados para a avaliação antropométrica infantil ${ }^{5,20}$.

Dentre os erros mais freqüentes observados na aferição da massa corporal destacou-se a não calibração da balança antes do procedimento e o fato de não se despir adequadamente as crianças antes que fossem pesadas, o que pode ter acarretado variações consideráveis na medida. Além disso, somente na metade das aferições realizadas os antropometristas aguardaram o alinhamento da agulha do braço e o fiel da balança antes da leitura, resultado semelhante ao observado entre os profissionais dos centros de saúde do município do Rio de Janeiro ${ }^{19}$, onde, todavia, os procedimentos referentes à "calibração da balança" e "despir a criança" foram realizados corretamente em proporções superiores (56,3\% e $71,5 \%$, respectivamente) àquelas aqui divulgadas.

De acordo com o coeficiente Kappa de Cohen, houve uma concordância substancial entre as classificações nutricionais segundo o índice peso-para-idade obtidas pelo pesquisador no equipamento padrão em relação às produzidas pelos antropometristas e, quando nessa comparação se substituiu a classificação obtida no serviço pela realizada pelo pesquisador usando o equipamento do serviço, houve uma concordância quase perfeita. Considerando a inexistência de erro na obtenção da informação relativa à idade da criança, esse procedimento avalia a confiabilidade da medida da massa corporal utilizada na composição do índice peso-para-idade. Os resultados obtidos indicam que os erros decorreram tanto por conta da técnica utilizada pelos antropometristas, como por problemas relativos aos equipamentos (balanças) existentes nos EAS.

Capelli et al. ${ }^{19}$, avaliando a qualidade do valor da medida de massa corporal de crianças menores de cinco anos atendidas nos centros de saúde do município do Rio de Janeiro, encontraram concordância perfeita $(K=1)$ entre as classificações nutricionais. Todavia, nesse estudo os equipamentos não se constituíram em objeto de avaliação, haja vista que foram submetidos à manutenção e aferição prévias. Adicionalmente, os pesquisadores não repetiram os procedimentos técnicos para obtenção da massa corporal, limitando-se a "ler" o valor da medida na escala da balança, imediatamente após o trabalho realizado pelo técnico local.

A concordância entre as classificações padrão e as obtidas pelos antropometristas em relação ao índice altura-para-idade foi considerada discreta, passando a moderada quando se comparou os resultados produzidos pelo pesquisador no equipamento padrão com aqueles produzidos pelo pesquisador usando o equipamento do serviço. Esses dados sugerem sérios problemas devidos à falta de capacitação e/ ou motivação dos funcionários, bem como à falta de adequação e/ou manutenção dos instrumentos utilizados. Conforme já referido, observou-se uma diversidade de instrumentos disponíveis no serviço de saúde para a realização das medidas de comprimento/altura, aliada ao mau posicionamento dos mesmos e à inadequação da técnica utilizada pelos antropometristas. Tal realidade compromete a qualidade das medidas antropométricas obtidas, o 
diagnóstico/classificação nutricional, e também a confiabilidade das informações produzidas nos serviços de saúde, as quais serão utilizadas na composição dos indicadores do Sistema de Vigilância Alimentar e Nutricional do Estado.

Conforme argumentos apresentados por Capelli et al..$^{19}$, existe uma certa limitação do teste de Kappa para aplicação com os fins ora utilizados, na medida em que é possível ocorrer uma alta concordância nas classificações, ainda que existam discrepâncias importantes nos valores individuais obtidos (baixa precisão). Isto pode ser explicado pelo fato de a classificação abranger, em cada situação (baixo peso, adequação e sobrepeso, para o índice peso-para-idade, por exemplo), uma ampla faixa de valores de massa corporal em cada categoria. Desta forma, ainda que haja variações relevantes entre os valores aferidos, não obrigatoriamente serão observadas alterações na classificação nutricional das crianças estudadas, caso tais erros ocorram de forma aleatória, pois o número de falsos positivos tenderia a ser anulado pelo de falsos negativos. Todavia, no nível individual, as crianças cujos valores de massa corporal forem sub ou superestimados podem ter o diagnóstico nutricional comprometido, especialmente aquelas que tiverem suas medidas reais nos pontos limítrofes dos intervalos da normalidade (percentis 3, 10 e 97).

De acordo com de Onis et al. ${ }^{7}$, o parâmetro mais comumente utilizado para avaliar a falta de precisão entre medidas repetidas é o ETM, que analisa a magnitude das diferenças entre as aferições padrão e aquelas em avaliação. Os resultados obtidos pelo cálculo do ETM em relação às variáveis peso e estatura ou comprimento nas diferentes situações em que foram obtidas e segundo faixas etárias $(</ \geq$ de 24 meses), demonstraram problemas relativos à técnica e aos equipamentos. Os valores obtidos pelo pesquisador no equipamento do serviço indicam que as balanças de plataforma encontram-se em condições adequadas de funcionamento; todavia, o resultado aparentemente satisfatório com relação aos estadiômetros (horizontal e vertical) deve ser analisado com cautela, visto que poucos EAS dispunham desse equipamento, comprometendo a avaliação.

Quanto aos testes de validade diagnóstica, em todas as situações houve alta especificidade, o que gera um alto valor preditivo negativo, mas, em contrapartida, um grande número de falsos negativos, ou seja, indivíduos portadores do agravo, porém, por terem sido equivocadamente diagnosticados como eutróficos, deixam de receber a atenção adequada por parte dos profissionais de saúde.

Verificou-se, por meio do teste dos sinais, que na comparação dos resultados "Padrão-ouro vs. antropometrista com equipamento do serviço" houve erro sistemático no sentido de superestimar o peso real da criança. Tal fato, evidentemente, reduz a chance do diagnóstico de desnutrição, razão pela qual se observou menor sensibilidade da classificação realizada no âmbito do serviço. Chama a atenção a elevada taxa de falsos negativos resultante desse erro: $38,5 \%$ para o peso-para-idade. O resultado prático dessa observação, considerando Alagoas como um todo, é a subestimativa da prevalência de desnutrição no Estado, além da falta de atenção adequada às crianças portadoras de desnutrição e não corretamente diagnosticadas. De acordo com os dados ora apresentados, a principal explicação para tal ocorrência seria a pesagem sem despir a criança, conforme está preconizado no manual de normas técnicas do Ministério da Saúde 5 .

Quanto ao índice altura-para-idade, assumindo-se que problemas decorrentes de falhas na fixação da fita métrica gerariam um erro aleatório, resta como explicação para o viés observado (maior freqüência de valores inferiores obtidos pelo pesquisador com o equipamento do serviço em relação às obtidas pelo padrão-ouro) a ausência de subdivisões em mm em proporção importante das fitas encontradas nos EAS, fazendo com que o observador buscasse a marcação (em centímetro) mais próxima ao ponto de tangência à cabeça. Todavia, é necessário 
um estudo específico para esclarecer essa hipótese.

$\mathrm{O}$ acompanhamento do crescimento figura entre as cinco ações básicas prioritárias normatizadas pelo Ministério da Saúde, visando incrementar a capacidade resolutiva dos serviços de saúde na atenção à criança ${ }^{6}$. Adicionalmente, o valor da massa corporal é muitas vezes utilizado para o cálculo da dosagem de determinados fármacos. Todos esses aspectos enfatizam a importância da obtenção precisa das medidas antropométricas no contexto dos serviços de saúde.

Diante do exposto conclui-se que a baixa confiabilidade observada para as variáveis comprimento e estatura desaconselha sua utilização na construção de indicadores de saúde até que investimentos sejam efetua- dos para reverter essa condição. Isso significa aquisição de estadiômetros pediátrico e vertical e a correspondente capacitação do corpo técnico para sua utilização.

Quanto à variável massa corporal, embora possa ser utilizada, é necessário melhorar sua qualidade por meio da capacitação dos antropometristas e da manutenção periódica das balanças.

Adicionalmente, recomenda-se instituir um programa de supervisão sistemática visando à solução de problemas locais, bem como efetivar um trabalho de motivação dos funcionários e padronização de condutas.

\section{Conflito de interesses: Não há.}

\section{Referências}

1. Brasil. Lei 8.080. Diário Oficial da União,19 de setembro de 1990

2. Silva DO, Engstrom EM, Zaborowski EL. SISVAN: instrumento para o combate aos distúrbios nutricionais na atenção à saúde - o diagnóstico coletivo. Rio de Janeiro: Editora Fiocruz; 2002.

3. Ministério da Saúde. Política nacional de alimentação e nutrição. 2a ed. Brasília: Ministério da Saúde; 2003.

4. Brasil. Lei 11.346. Diário Oficial da União de 18 de setembro de 2006.

5. Ministério da Saúde. Vigilância alimentar e nutricional SISVAN: orientações básicas para a coleta, processamento, análise de dados e informação em serviços de saúde. Série A. Normas e Manuais Técnicos. Brasília: Ministério da Saúde; 2004. Disponível em http://nutricao. saude. gov.br/documentos/orientacoes_basicas_sisvan.pdf. [Acessado em 06 de janeiro de 2009]

6. Ministério da Saúde. Saúde da criança: acompanhamento do crescimento e desenvolvimento infantil. Série Cadernos de Atenção Básica; n. 11. Série A. Normas e Manuais Técnicos. Brasília: Ministério da Saúde; 2002.

7. de Onis M, Garza C, Victora CV, Onyango AW, Frongillo EA, Martines J, for the WHO Multicentre Growth Reference Study Group. The WHO Multicentre Growth Reference Study: planning, study design, and methodology. Food Nutr Bull 2004; 25(1)(S1): 15-26.
8. Carvalho MF, de Lira PI, Romani S de A, Santos IS, Veras AA, Batista Filho M. Acompanhamento do crescimento em crianças menores de um ano: situação nos serviços de saúde em Pernambuco, Brasil. Cad Saúde Pública 2008; 24(3): 675-85.

9. Ministério da Saúde. Cadastro Nacional de Estabelecimentos de Saúde. Disponível em http:// tabnet. datasus.gov.br/cgi/deftohtm.exe?cnes/cnv/estabAL.def. [Acessado em 06 de janeiro de 2005]

10. Habicht JP. Estandarizacion de métodos epidemiológicos cuantitativos sobre el terreno. Bol Oficina Sanit Panam 1974; 76(5): 375-84.

11. National Center for Health Statistics (NCHS). Growth curves for children birth-18 years. Vital and Health Statistics Series 11, n.165 DHEW Publ. Washington DC: U.S. Printing Office; 1977.

12. Landis JR, Koch GG. The measurement of observer agreement for categorical data. Biometrics 1977; 33: 15974 .

13. Ulijaszek SJ, Kerr DA. Anthropometric measurement error and the assessment of nutritional status. Br J Nutr 1999; 82(3):165-77.

14. Pederson D, Gore C. Error en la medición antropométrica. In: Norton K, Olds T (eds). Antropométrica. Argentina: Biosystem Servicio Educativo; 2000. 
15. Daniel WW. Some basic probability concepts. In: Daniel WW. Biostatistics: a foundation for analysis in the health sciences. New York: John Wiley; 2005.

16. Cuervo MR, Aerts DR, Halpern R. Vigilância do estado nutricional das crianças de um distrito de saúde no Sul do Brasil. J Pediatr 2005; 81: 325-31.

17. Batista Filho M, Romani SAM, editores. Atenção à saúde materno-infantil no Estado de Pernambuco. Recife: Editora Bagaço; 2000.

18. Veras AACA, Osório MM, Frias PG, Sarinho SW, Romani SAM. Avaliação da atenção à saúde da criança e da gestante em serviços municipais de saúde em Recife. Série Publicações Científicas do Instituto Materno Infantil de Pernambuco. Recife: Instituto Materno Infantil de Pernambuco; 2004.
19. Capelli JCS, Anjos LA, Castro IRR. Qualidade do valor da medida de massa corporal nos Centros Municipais de Saúde do Município do Rio de Janeiro, 1996. Cad Saúde Pública 2002; 18(1): 63-70.

20. Monego ET, Menezes IHCF, Peixoto MRG, Gil MF, Barbosa LS, Vieira EC. Antropometria: manual de técnicas e procedimentos - vigilância nutricional. $3^{\mathrm{a}} \mathrm{ed}$. Goiânia: CECAN-RCO; 2007.

Recebido em: 07/01/09

Versão final reapresentada em: 29/10/09

Aprovado em: 30/11/09 